\title{
Justification ou bon pour des prestations
}

\section{Jürg Unger-Köppel}

Dr méd., membre du Comité central de la FMH, responsable du département Médecine et tarifs hospitaliers

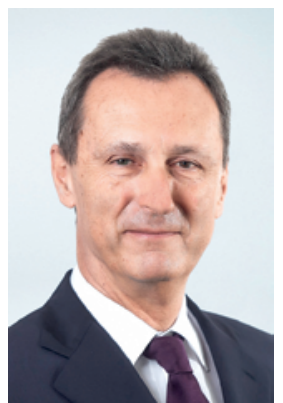

Lors du Forum sur les DRG, un orateur plus âgé venu d'Allemagne déclarait: «Par le passé, un contrat non écrit existait dans notre société: les médecins s'occupaient de nous et nous veillions sur eux. Mais ce contrat avec les médecins n'existe plus aujourd'hui.» Les conséquences de cette résiliation de contrat par la société sont bien perceptibles: lorsqu'on est assis à la même table que ceux qui remboursent les prestations, la méfiance l'emporte sur notre activité de médecin et, par conséquent, les prestations fournies doivent être justifiées de manière toujours plus détaillée. Le résultat: plus de 1000 DRG, d'innombrables CHOP, environ 2500 positions dans le TARDOC et, parallèlement, la saisie des prestations qui empiète sur notre temps avec les patients. Le soir, au lieu de nous rendre à leur chevet pour un peu de réconfort et de soutien, nous restons devant nos systèmes de comptabilité et notons en détail les minutes de prestations dispensées dans la journée à chaque patient pour que la prochaine version des DRG devienne encore meilleure. Nous rentrons ensuite fatigués à la maison, tard le soir, et contrôlons à notre tour la facture du garagiste.

Avec le tarif de la réadaptation (ST Reha), la justification des prestations prendra le pas sur les forfaits journaliers.

Si les forfaits journaliers nécessitent un temps de saisie minimal (patient présent: oui/non), ils n'ont plus cours en psychiatrie depuis 2018. La réadaptation reste donc aujourd'hui le seul domaine dans lequel il suffit de justifier la présence du patient pour les obtenir. Il est bien évident que l'indication pour la réadaptation hospitalière doit avoir été justifiée au préalable de manière convaincante sur la base de l'état du patient et de son potentiel de rééducation. Mais ce qui est fait jour après jour pour le patient en réadaptation n'a pas besoin d'être montré, du moins pas encore, pour être pris en charge par les caisses-maladie. Ainsi, figurer sur la liste hospitalière, c'est comme obtenir un bon pour des prestations sans qu'on en connaisse la valeur exacte.

\section{Conjointement avec $\mathrm{H}+$, la FMH a obtenu que} le temps de saisie ne soit pas excessif.

A l'image de la méfiance envers le corps médical qui règne actuellement en politique, le futur tarif de la réadaptation (ST Reha) exige lui aussi que les prestations soient justifiées, ouvrant ainsi le débat sur les CHOP et la représentativité exacte des coûts. Ceux qui veulent que le système soit aussi simple que possible se confrontent rapidement à ceux qui veulent une saisie aussi précise que possible - deux objectifs diamétralement opposés qui se retrouvent dans tous les systèmes tarifaires.

Conjointement avec les sociétés de discipline concernées, la division Médecine et tarifs hospitaliers de la FMH a planché pendant plus de deux ans sur les prestations de base et les prestations supplémentaires en réadaptation et les a soumises aux partenaires de SwissDRG SA et à l'Office fédéral de la statistique (OFS) en vue de leur introduction dans la CHOP. Au final, la FMH a réussi à convaincre ses partenaires de suivre une voie médiane en guise de compromis. Cette voie exige un certain temps pour la saisie tout en essayant de le limiter (cf. l'article de B. Trezzini et B. Meyer dans le BMS 2019;100(6):161-162). Fin avril 2019, la position commune des partenaires de négociation a été réaffirmée lors d'une réunion à l'OFS. Les caisses et les cantons ont réclamé très clairement la transparence en ce qui concerne les prestations fournies tandis que la $\mathrm{FMH}$ a obtenu, avec le soutien de $\mathrm{H}+$, que le temps de saisie ne soit pas excessif. 